Abschluss der Testwochen

Deutliche Hilfe

bei Zahnfleischproblemen

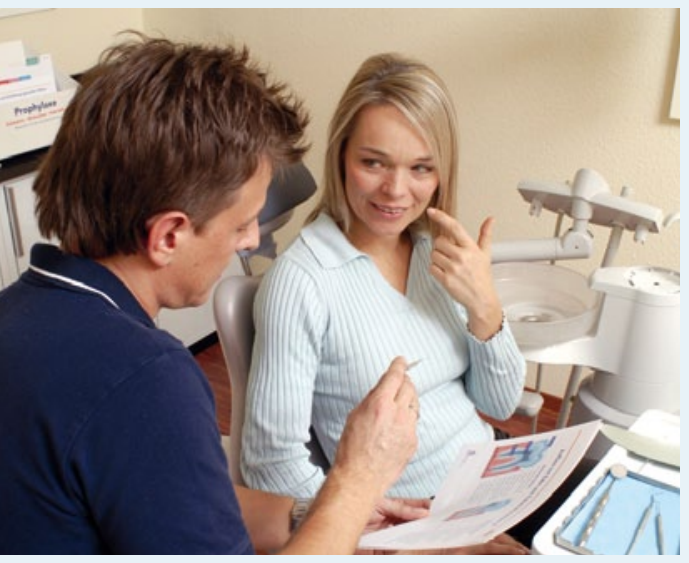

Die Ergebnisse der meridol ${ }^{\circledR}$ Testwochen sprechen für sich: Bereits nach kurzer Zeit gingen die Zahnfleischprobleme der Testerinnen und Tester erkennbar zurück. Deshalb würden fast sämtliche Teilnehmer die ausprobierten Produkte weiterverwenden und weiterempfehlen.

Fast 1800 Patienten mit Zahnfleischproblemen haben sich an der Aktion beteiligt. Drei Wochen lang testeten sie die Produkte des meridol ${ }^{\circledR}$ Verbundes, bestehend aus Zahnpasta, Mundspülung und Zahnbürste. Zum Schluss hielten sie ihre Erfahrungen in einem Fragebogen fest. Die Resultate sind eindeutig: Bei über 90\% der Teilnehmer mit Zahnfleischproblemen gingen diese im Laufe der Testzeit vollständig zurück. 65\% der Testerinnen und Tester bescheinigten dabei bereits nach 1-wöchiger Anwendung eine deutliche Verbesserung ihrer Beschwerden. Aufgrund dieser guten Beurteilung kann nicht verwundern, dass $96 \%$ der Testpersonen nicht nur bereit sind, die Produkte weiterzuverwenden, sondern auch weiterzuempfehlen.

Die starken Resultate des Patiententests bestätigen laut Hersteller GABA eindrucksvoll die in mehr als 150 Studien belegte spürbare Wirksamkeit der meridol ${ }^{\circledR}$-Produkte mit ihrer einzigartigen Wirkstoffkombination Aminfluorid/ Zinnfluorid. Das Unternehmen bedankt sich bei allen Praxen, die die Aktion unterstützt haben.

Nach einer Pressemitteilung der

GABA GmbH, Lörrach

E-Mail: info@gaba.com

\section{Dr. Steffi Trosse erhielt „DGK-Praktikerpreis ${ }^{\circ}$ Behutsam Ångste abgebaut}

Dr. Steffi Trosse, Berlin, ist die Preisträgerin des „DGK-Praktikerpreises“ 2012. Die renommierte Auszeichnung, gestiftet von der Deutschen Gesellschaft für Kinderzahnheilkunde (DGK) und dem Mundpflegehersteller GABA, wird jährlich für herausragende Forschungsarbeiten aus dem Bereich der kinderzahnheilkundlichen Praxis vergeben. Er ist mit 1500 Euro dotiert. Die Preisträgerin erhielt diese Auszeichnung für eine Fallpräsentation, die sie auf der DGK-Tagung des vergangenen Jahres in Halle/ Saale gehalten hatte. Dort beeindruckte sie ihr Publikum durch eine Videodokumentation über die Behandlung eines 10-jährigen Jungen mit generalisierter Angststörung. Konnte der Junge bislang nur unter Vollnarkose behandelt werden, gelang es der Zahnärztin mit viel Geduld und kleinen Schritten in der Verhaltensführung, die Mitarbeitsfähigkeit bei dem betroffenen Kind herzustellen. Durch schonendes Vorgehen und ein konsequentes Individual-Prophylaxe-Programm gelang es Dr. Trosse, die Mundgesundheit des Jungen zu sta-

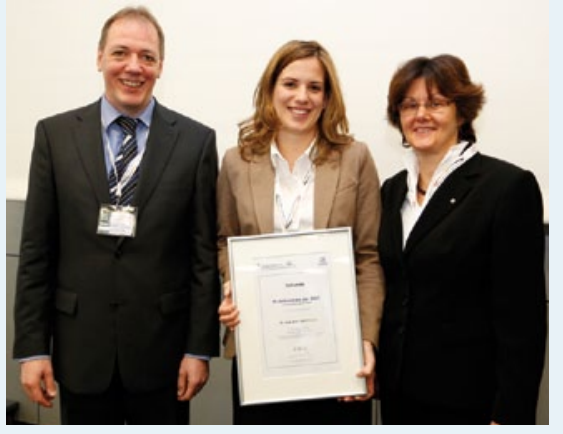

Preisträgerin Dr. Steffi Trosse (m.) mit Prof. Christian Hirsch und Dr. Vanessa de Moura Sieber (GABA).

bilisieren und ihm weitere Narkosebehandlungen zu ersparen.

Dr. Steffi Trosse ist in der Berliner Zahnärzte- und Prophylaxepraxis „Zeit für schöne Zähne“ von Dr. Matthias Thuma tätig. Dort ist sie - als zertifizierte Absolventin des Curriculums Kinderzahnheilkunde der DGK - auf Kinder- und Jugendzahnheilkunde spezialisiert.

Dieser Beitrag ist entstanden mit freundlicher Unterstützung der

GABA GmbH, Lörrach.

\title{
Karies bei Risikopatienten \\ Herausforderung für die Praxis
}

„Strategien zur Prophylaxe bei Patienten mit hohem Kariesrisiko - Bewährtes und Neues" lautete der Titel einer Fortbildungsveranstaltung der GABA in der Kulturfabrik Haldensleben (Sachsen-Anhalt). 70 Zahnärzte, Prophylaxefachkräfte und Zahnmedizinische Fachangestellte verfolgten die Vorträge der Referentinnen. Oberärztin

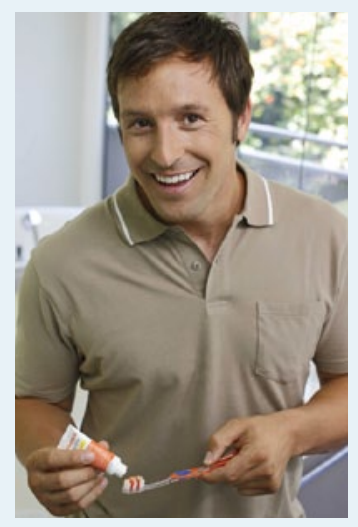
dierung ein Leben lang Wie geht das?". Für die Teilnahme gab es 4 Fortbildungspunkte.

GABA bietet mehrere Produkte zur Intensivfluoridierung an, unter anderem elmex ${ }^{\circledR}$ gelée mit Aminfluorid. Darüber hinaus ist Aminfluorid, das als besonders wirksam gilt, auch in vielen Zahnpasten und Zahnspülungen des Unternehmens enthalten. Als

PD Dr. Katrin Bekes (Universität Halle) referierte über „MolarenInzisiven-Hypomineralisation - Was müssen wir wissen?“. Anschließend beleuchtete Oberärztin Dr. Leonore Kleeberg (Zahnärztekammer Sachsen-Anhalt) das Thema „Motivation und Prophylaxe - Ein Muss für jeden KFO-Patienten“. Zum Abschluss gab Dr. Vanessa de Moura Sieber (GABA) Tipps zur „Intensivfluori-
Herzstück der häuslichen Prophylaxe gilt seit Jahrzehnten der Doppel-Schutz aronal ${ }^{\circledR} /$ elmex $^{\circledR}$, der im März moderat überarbeitet wurde.

Einen ausführlichen Bericht zur Veranstaltung in Haldensleben gibt es im Internet: www.gaba-dent.de/fortbildung.

Nach einer Pressemitteilung der GABA GmbH, Lörrach 